\title{
Effect of wettability and surface roughness on ice-adhesion strength of hydrophilic, hydrophobic and superhydrophobic surfaces
}

\author{
T. Bharathidasan, Vijay Kumar", M.S. Bobji ${ }^{\#}$, R.P.S. Chakradhar*, Bharathibai J. Basu* \\ Surface Engineering Division, CSIR- National Aerospace Laboratories, Bangalore - 560017, India. \\ \# Department of Mechanical Engineering, Indian Institute of Science, Bangalore - 560003, India.
}

\begin{abstract}
The anti-icing properties of hydrophilic, hydrophobic and superhydrophobic surfaces/coatings were evaluated using a custom-built apparatus based on zero-degree cone test method. The ice-adhesion reduction factor (ARF) of these coatings has been evaluated using bare aluminium alloy as a reference. The wettability of the surfaces was evaluated by measuring water contact angle (WCA) and sliding angle. It was found that the ice-adhesion strength $(\tau)$ on silicone based hydrophobic surfaces was $\sim 43$ times lower than compared to bare polished aluminium alloy indicating excellent anti-icing property of these coatings. Superhydrophobic coatings displayed poor anti-icing property in spite of their high water repellence. Field Emission Scanning Electron Microscope reveal that Silicone based hydrophobic coatings exhibited smooth surface whereas the superhydrophobic coatings had a rough surface consisting of microscale bumps and protrusions superimposed with nanospheres. Both surface roughness and surface energy play a major role on the ice-adhesion strength of the coatings. The 3D surface roughness profiles of the coatings also indicated the same trend of roughness. An attempt is made to correlate the observed $\tau$ of different surfaces with their wettability and surface roughness. It was concluded that smooth surface with low surface energy are responsible for low ice-adhesion strength.
\end{abstract}

Keywords: Anti-icing; ice-adhesion strength; hydrophobic; superhydrophobic; water contact angle; surface roughness

* Corresponding authors : E-mail address: bharathijbasu@gmail.com (Bharathibai J. Basu); chakra@nal.res.in (R.P.S. Chakradhar) Tel.: +91 080 25086498; Fax: +91 08025210113 


\section{Introduction}

The ice accumulation on solid surfaces during cold weather in cold regions causes serious problems on power lines, telecommunication networks, radars, aircrafts, helicopters, ships, wind turbines because of safety and energy efficiency considerations [1-4]. Especially when ice builds-up on aircraft and gas turbine engine structures has been disastrous in the aircraft industry. When ice builds up on the wings or leading edges of airplanes, it increases drag and decreases lift. Thus the presence of ice can adversely affect the aerodynamic performance of airfoils, particularly wings, fan blades. Therefore, prevention and control of ice accumulation on structures have important applications. In the past, several anti-icing systems have been developed to reduce the ice formation on structures [2-4]. The commonly used ice removal strategies have several drawbacks mainly due to the detrimental environmental consequences with freeze retarding chemicals and energy consumption for heating. A common strategy for minimizing ice formation and adhesion has been the application of ice-phobic coatings which can be either sacrificial or permanent [2]. Sacrificial coatings like silicone greases are removed from substrates gradually, requiring periodic application [3]. Sol-gel materials that slowly release freezing point depressants also belong to this category [4]. On the other hand, permanent hydrophobic coatings such as polymers reduce the strength of ice-adhesion to surfaces and they do not require periodic reapplication [5-9]. The ice-phobic coatings have been proposed as passive technique to prevent or reduce the ice accumulation. These coatings can provide reduced ice-adhesion or delayed water freezing on their surfaces which results in lower ice accumulation on such coated surfaces [9-14].

It is commonly believed that superhydrophobic (SH) surfaces with high water repellence would have weak ice-adhesion strength. SH surface can be fabricated by creating surface texture with hierarchical micro-nano roughness and by controlling the chemical 
composition using low surface energy materials. Such artificial SH surfaces have been reported to delay and reduce the accumulation of wet snow, ice, or frost and hence these coatings have demonstrated high potential in the anti-ice coating sector [9-23]. Saito et al [9] reported the possible anti-icing applications of SH coatings for the first time. Later, several groups also have observed reduced ice accumulation on $\mathrm{SH}$ coatings and studied the correlation between the superhydrophobicity and decrease in ice-adhesion [10-23]. Some of them have reported reduced ice-adhesion for SH coatings [10-18], while some have emphasized on the effect of contact angle hysteresis (CAH) on ice-adhesion strength $[5,6,15,16]$. Recently, Meuler et al $[5,6]$ have reported a linear relationship between ice-adhesion strength and the water wettability parameter $\left[1-\cos \theta_{\text {rec }}\right]$, where $\theta_{\text {rec }}$ is the receding contact angle of water. Tourkine et al [17] and He et al [20] reported delayed water freezing on rough SH surfaces. Cao et al [10] and Wang et al [18] reported low ice accumulation on SH surfaces exposed to "freezing rain" conditions. Though both reduced ice-adhesion strength and delayed ice accretion on SH surfaces have been reported, their antiice performance under real atmospheric icing conditions has not yet been adequately studied.

Recently, there have been controversial reports on the anti-icing performance of SH surfaces [24-29]. Varanasi et al [24] have reported that frost formation inside the textures of SH surfaces could increase the ice-adhesion. Later, other researchers also have supported their findings [25-29]. Kulinich et al [26] have shown that anti-icing efficiency of SH surfaces is significantly lower in a humid atmosphere. Very recently, Chen et al [29] have shown that SH surfaces cannot reduce ice-adhesion, and the ice adhesion strength on $\mathrm{SH}$ surface and superhydrophilic surface were almost the same. As on today it is not clear the effect of surface roughness, which is believed to be affecting the ice-repelling performance, has not been adequately studied. As a part of our programme on anti-icing coatings, in the 
present work, we have studied the ice-adhesion strengths of coatings with different wettabilities, namely hydrophilic polyurethane and PMMA, hydrophobic silicones, and superhydrophobic silicone and PMMA-based nanocomposite coatings. Even though several methods have been developed to measure ice-adhesion strength, some of them based on freezing of the super-cooled water drops and delayed ice accretion may not be suitable for simulating the atmospheric icing conditions and prolonged contact of ice with the surfaces. Therefore, custom-built instrument based on zero-degree cone method was used for evaluating ice-adhesion strength of these surfaces and the results were correlated to the combined effect of wettability and surface roughness.

\section{Experimental}

\subsection{Materials}

The starting materials used in the present study were two-component polyurethane (PU) paint which was procured from Southfield paints, Bangalore. Polymethylmethacrylate (PMMA) (molecular weight -350000) was procured from Alfa Aesar. Silicone elastomer, R2180 and CF1-135 primer were procured from Nusil technology LLC, Carpinteria. Silicone resin, RTV11 and silicone primer SS 4044 were procured from GE Bayer Silicones. Hydrophilic fumed silica (Cab-O-Sil EH5, particle size of about 10-15 nm) was procured from Cabot Sanmar Ltd. Hydrophobically modified fumed silica was procured from ABCR GmbH \& Co, Germany. Dibutyl tin dilaureate (DBT) was procured from Aldrich. The solvents used in the present work were toluene and xylene and are procured from Merck India.

\subsection{Preparation of coatings}

All the coatings under investigation were prepared by spraying the solutions of polymers, paints and precursor mixtures on Al substrate and glass slides. The coatings were 
applied on an aluminium alloy (AA) cylinders of $2.5 \mathrm{~cm}$ dia and $9.0 \mathrm{~cm}$ length for evaluation of ice-adhesion strength and on $\mathrm{Al}$ coupons of $5 \mathrm{~cm}$ x $5 \mathrm{~cm}$ size for WCA measurements and other characterizations. Prior to the application of the coatings, the substrates were cleaned with toluene. A spraygun with nozzle size of $1 \mathrm{~mm}$ dia and compressed air pressure of 20-25 p.s.i were used for spraying the solutions.

Two hydrophilic coatings were prepared using PU and PMMA. PU solution was prepared by mixing polyurethane base and hardener in 2: 1 ratio. PMMA solution $(2.0 \mathrm{wt} \%)$ was prepared by dissolving the polymer in toluene. PU and PMMA coatings were cured at room temperature. Two types of silicone hydrophobic coatings were prepared using commercial silicones like R2180 and RTV 11. R2180 solution was prepared by mixing equal volumes of parts A and B of R2180 with xylene. The mixture was magnetically stirred for 20 minutes and kept in vacuum to remove air bubbles before spraying onto aluminium alloy (AA) substrates applied with CF1-135 primer. RTV11 solution was prepared by mixing $4.0 \mathrm{~g}$ resin in about $10 \mathrm{ml}$ toluene and then dispersed well using glass rod for about 10 minutes. It is mixed with $4 \mathrm{ml} 4 \mathrm{wt} \%$ DBT solution as a catalyst before spraying on aluminium substrates applied with SS 4044 primer.

In addition to the hydrophilic and hydrophobic coatings, three different types of superhydrophobic polymer-silica nanocomposite coatings (namely R2180-EH5, RTV11-EH5 and PMMA-HMS) were selected for the present study. R2180-EH5 was prepared by mixing a solution of equal volumes of parts A and B of R2180 resin in xylene with ultrasonically dispersed cabosil EH5 such that the silica to resin weight ratio was 1:5. Similarly, RTV11EH5 was prepared by mixing RTV11 solution in toluene with ultrasonically dispersed cabosil EH5 with silica to resin weight ratio of $1: 5$ and $4 \mathrm{wt} \%$ DBT and stirring the mixture for 15 min. The R2180-EH5 and RTV11-EH5 mixtures were sprayed onto aluminium alloy substrates applied with CF1-135 and SS4044 primers respectively. R2180 and R2180-EH5 
coatings were cured at room temperature for overnight, then heated at $75^{\circ} \mathrm{C}$ for 45 minutes and at $150^{\circ} \mathrm{C}$ for $2 \mathrm{~h} 15$ minutes. RTV11 and RTV11-EH5 coatings were cured at room temperature for $24 \mathrm{~h}$. PMMA-HMS coatings were prepared by mixing PMMA solution (2 wt $\%$ ) with ultrasonicated hydrophobically modified silica (HMS) so that the weight ratio of HMS to PMMA was 5:1. The mixture was magnetically stirred for about 30 min and sprayed onto clean AA substrates. The coated specimens were cured at room temperature for $24 \mathrm{~h}$.

\subsection{Characterization}

Water contact angles (WCA) of the coatings were measured using a contact angle analyser (model Phoenix 300 Plus from M/s Surface Electro Optics, South Korea). Measurements were made using tangent line-fitting mode. Water sliding angle (SA) was measured using a home-made instrument. The drop volume for WCA and SA measurements was $8 \mu \mathrm{L}$. Deionised milli Q water (surface tension: $72.99 \mathrm{mN} / \mathrm{m}$ ) was used for measurements. An average of five measurements was taken for reporting WCA and the error in measurements were within $\pm 2^{\circ}$. The thickness of the coatings was measured by surface profilometry and is found to be in the range10 - $12 \mu \mathrm{m}$. The surface morphology of the coatings was studied using Field Emission Scanning Electron Microscope FESEM (model Carl Zeiss Supra 40). The3D roughness profiles were measured using 3D profilometer (model Nano Map 500LS from AEP Technology, USA).

The ice-adhesion strength of the prepared coatings was tested using a custom-built instrument based on zero-degree cone test method as shown in Fig. 1. In this method, the coating is applied on aluminium alloy AA6061cylinder and the ice is formed in an annular space between the test surface and a standard surface by keeping the coated cylinder assembly with jacket in a deep freezer maintained at $-20^{\circ} \mathrm{C}$ for $24 \mathrm{~h}$. The coated test cylinder was placed in the specimen holder and pushed outward using a motor-driven cross head at 
slow speed and the breakage strength is measured using a load cell. The drive and record switches are turned off after the maximum load is reached. Ice-adhesion strength $(\tau)$ is the shear stress at which the frozen water droplet became detached from the sample surface as indicated by sudden drop of the sensed shear force to zero and is calculated as the ratio of the maximum load and the contact area. The durability of the coatings was evaluated by repeated measurements of $\tau$ or by measuring $\tau$ after subjecting the coated substrates to six icing deicing cycles at $-20^{\circ} \mathrm{C}$ for $24 \mathrm{~h}$.

\section{Results and Discussion}

\subsection{Wettability and ice-adhesion strength of the coatings}

Wettability of the solid surface is very important for the study of ice-adhesion strength of the coatings. Table 1 lists the water contact angle and sliding angle of the hydrophilic, hydrophobic and superhydrophobic coatings studied in the present work. The WCA of PU and PMMA were $76 \pm 1.5^{\circ}$ and $73 \pm 1.2^{\circ}$ respectively indicating the hydrophilic nature of these surfaces. Bare aluminium alloy surface also was hydrophilic with WCA of 67 $\pm 2^{\circ}$. The WCA of R2180 and RTV11 were $103 \pm 2.2^{\circ}$ and $105 \pm 1.5^{\circ}$ respectively which clearly showed that these surfaces were hydrophobic. On the other hand, the nanocomposite coatings like R2180-EH5, RTV11-EH5 and PMMA-HMS were superhydrophobic with WCA of $>150^{\circ}$ and water drops rolled on their surface with $\mathrm{SA}<2^{\circ}$. It was seen that the hydrophobic R2180 and RTV11 coatings were transformed into superhydrophobic surfaces with the incorporation of appropriate amounts of silica nanoparticles. Fig. 2 shows images of water droplet on bare AA and some of the typical coatings like PU, hydrophobic silicone coating, RTV11 and a typical superhydrophobic coating like RTV11-EH5. 
A surface having high wettability tends to allow the water drop to spread over a relatively wide area thereby wetting the surface. The work of adhesion $(W)$ on the surfaces can be calculated by using Young-Dupre's equation $[26,29,30]$

$$
\mathrm{W}_{\mathrm{sl}}=\gamma_{\mathrm{la}}(1+\cos \theta)
$$

where $\mathrm{W}_{\mathrm{sl}}$ is the work of adhesion between solid and liquid surface and $\gamma_{\mathrm{la}}$ is the liquid-air interfacial surface tension. The values obtained for work of adhesion calculated using equation (1) are included in Table 1. It is observed that the work of adhesion follow the relation bare aluminium alloy $>$ PMMA $>$ PU $>$ R2180 $>$ RTV11 $>$ PMMA-HMS $>$ R2180EH5 > RTV11-EH5 indicating that superhydrophobic coatings had the lowest work of adhesion.

Figures 3 and 4 show the load versus displacement plots for bare aluminium alloy and the coatings and the maximum load can be obtained from these plots. Ten measurements of ice-adhesion strengths were made for each coating and the average $\tau$ values with standard deviation were calculated and are shown in Table 2. It is observed that the ice-adhesion force and displacement followed a linear relationship until the force suddenly dropped to zero indicating ice detachment. This suggests a linear elastic deformation followed by brittle failure. It was found that the hydrophilic PU and PMMA coatings have high ice-adhesion strength of 820 and $1575 \mathrm{kPa}$ respectively. The ice-adhesion strength of PU coating was slightly lower than that of bare AA $(1072 \mathrm{kPa})$ whereas $\tau$ of PMMA was higher than that of bare AA. Hydrophobic R2180 and RTV11 coatings were found to have low ice-adhesion strengths of $42 \pm 10 \mathrm{kPa}$ and $25 \pm 9 \mathrm{kPa}$ respectively. The ice-adhesion strength of $\mathrm{R} 2180$ coating was in agreement with the reported value of $37 \mathrm{kPa}$ by Sivas et al [7]. The RTV11 
coating has shown a lower $\tau$ value than that of R2180. Thus a comparison of hydrophilic and hydrophobic coatings showed that $\tau$ decreased with increase in WCA.

It is observed that the ice-adhesion strength for all the three $\mathrm{SH}$ coatings were found to be higher than that of hydrophobic silicone coatings. In the case of SH coatings like R2180-EH5 and RTV11-EH5, ice-adhesion strength was about $250 \pm 20 \mathrm{kPa}$ which was higher than that of the corresponding hydrophobic coatings, R2180 and RTV11. The ARF values were 25 and 43 for hydrophobic coatings like R2180 and RTV11 respectively whereas ARF was about 4 for the corresponding superhydrophobic coatings, R2180-EH5 and RTV11EH5. The ice-adhesion strength was very high $(800 \mathrm{kPa})$ for superhydrophobic PMMA-HMS which was similar to that of the hydrophilic PU coating but lower than pristine PMMA coating. Since SH coatings are highly water repellent, they are expected to have high ice repellence also. A few researchers have reported reduced ice-adhesion for SH coatings [5-23]. Low ice accumulation on SH surfaces exposed to natural and artificial "freezing rain" conditions have also been reported [10,18]. Delayed water freezing has been observed on rough SH surfaces $[17,20]$. Momen et al [23] reported that freezing time was considerably improved for SH surfaces compared to those obtained by RTV coating on polished Al surfaces. Their study showed an important reduction in WCA and increase in contact angle hysteresis for SH surface at cold temperatures below $0^{\circ} \mathrm{C}$. However, Varanasi et al [24] have reported that SH surfaces can exhibit increased ice-adhesion due to frost formation inside the textures. Kulinich et al [26] have shown that anti-icing efficiency of SH surfaces is significantly lower in humid atmosphere. Very recently, Zou et al [28] and Chen et al [29] also shown that SH surfaces cannot reduce ice-adhesion. In the present work, the authors have also obtained higher ice adhesion strength for SH coatings. This may be due to the increase of the contact area of ice and the rough microstructure of SH coating which may result in an anchoring effect with ice and coating getting embedded in each other. 
Ice-adhesion strengths of surfaces also depend on the method used for the measurement. Different $\tau$ values on bare aluminium alloy have been reported [11-14, 24]. The ice-adhesion strength of bare aluminium alloy was about $500 \mathrm{kPa}$ by centrifuge adhesion test (CAT), $1540 \mathrm{kPa}$ by tensile test and $1210 \mathrm{kPa}$ by zero cone test methods. The ice-adhesion strength values of the coatings were compared with that of bare aluminium alloy and $\operatorname{ARF}$ ( $\tau_{\text {Bare }} / \tau_{\text {coating }}$ ) values for each coating were calculated and are included in Table 2. The ARF for R2180 and RTV11 coatings were 25 and 43 respectively. Thus maximum ARF was obtained for RTV11 coatings indicating good icephobic property. The ARF value (43) of RTV11 was found to be much higher than the reported values for PTFE-based coatings by Farzaneh et al [11-14].

\subsection{Surface microstructure and surface roughness of the coatings}

It is well known that wettability is affected by surface microstructure, roughness and surface free energy. The surface morphology of the coatings was examined by using FESEM. Fig. 5 shows the FESEM images of R2180 and R2180-EH5 nanocomposite coatings. Hydrophobic R2180 coating exhibited smooth surface with wavy patterns. FESEM image of the superhydrophobic R2180-EH5 coating showed a rough surface morphology consisting of microscale bumps and protrusions superimposed with nanospheres. Therefore, the micronano binary rough structure obtained by incorporation of cabosil EH5 silica nanoparticles in R2180 silicone matrix transformed the wettability of the surface from hydrophobic to superhydrophobic state. Fig. 6 shows the FESEM images of the superhydrophobic RTV-EH5 nanocomposite coatings. The hydrophobic RTV11 coating was smoother than R2180 coating whereas RTV11-EH5 was very rough with protrusions of cabosil EH5 silica nanoparticle aggregates. The roughness of SH coatings prepared by spraying was dependent not only on 
the composition of the mixture but also on the spraying parameters. After the incorporation of silica nanoparticles in R2180 and RTV11 matrix, the coatings showed enhanced roughness which allows the formation of air pockets between water and the rough surface leading to composite solid liquid air interface. In order to get a better idea of the surface roughness of the different coatings, 3D surface roughness images of hydrophilic and hydrophobic coatings were examined and these are shown in Fig. 7. The 3D surface roughness images of the three superhydrophobic coatings are shown in Fig. 8. The surface roughness parameters of these coatings are listed in Table 3.

In general, the hydrophilic and hydrophobic coatings have lower average roughness $\mathrm{R}_{\mathrm{a}}$ and rms roughness $\left(\mathrm{R}_{\mathrm{q}}\right)$ whereas the roughness was high for $\mathrm{SH}$ coatings. The $\mathrm{R}_{\mathrm{a}}$ of PMMA was higher than that of PU coating. This higher roughness of hydrophilic PMMA coating may be the reason for the high ice-adhesion strength value obtained for PMMA. A surface with more polar groups would have larger ice-adhesion strength because of the stronger hydrogen bonds. PMMA surface may have more polar groups than PU surface leading to larger surface energy and larger ice-adhesion. As a result, the ice-adhesion strength of PMMA was the largest. Similarly, $\mathrm{R}_{\mathrm{a}}$ of R2180 was higher than that of RTV11 coating. The higher roughness of hydrophobic R2180 coating may result in higher $\tau$ value. Yang et al also have studied the ice-adhesion strength of fluoropolymers by tensile test method and their results showed that the pristine PTFE has the lowest tensile strength of $110 \mathrm{kPa}$ and it increased to $210 \mathrm{kPa}$ when the roughness of PTFE was enhanced by sand-blasting [25]. They suggested that the ice-adhesion strength is associated with both surface chemical composition and surface roughness. In the case of superhydrophobic coatings, surface roughness was high for all three coatings. $\mathrm{R}_{\mathrm{a}}$ was in the range of $1.5-2.0 \mu \mathrm{m}$. Surface energy of the polymer matrix was low for R2180-EH5 and RTV11-EH5 whereas it was high for PMMA matrix. This may be the reason for the higher $\tau$ of superhydrophobic PMMA-HMS of about $800 \mathrm{kPa}$ 
compared to that of R2180-EH5 and RTV11-EH5 superhydrophobic coatings. Thus the ice-adhesion strength is associated with both surface chemical composition and surface roughness. Yang et al [25] have concluded from their study of smooth and roughened PTFE coatings, that the smooth surface with low surface energy is responsible for low ice-adhesion strength. Cao et al [10] reported that the anti-icing capability of the superhydrophobic polymer-composite coatings not only depends on their superhydrophobicity but also on the size of the particles exposed on the surface and the critical size of the particles. In the present work, it was observed that the rough superhydrophobic coatings have different values of ice-adhesion strength indicating that the surface energy of the matrix is more important than their surface roughness.

Sivas et al [7] have obtained ice-adhesion strength of $37 \mathrm{kPa}$ for R2180 silicone elastomer coating by zero degree cone test. In the present study, lower ice-adhesion strength of $25 \mathrm{kPa}$ was obtained for RTV11 silicone coating. Another advantage of RTV11 coating is that it can be cured at room temperature whereas heat treatment at $135^{\circ} \mathrm{C}$ is required to cure R2180. It is also difficult to spray R2180 solution and get a smooth coating. Wang et al [18] reported that RTV silicone rubber coated aluminium substrate showed less ice accumulation compared to uncoated substrate at $-20^{\circ} \mathrm{C}$. Fig. 9 shows a comparative chart of the ice-adhesion reduction factor (ARF) with reference to bare AA for the coatings studied in the present work. From the chart it can be observed that ARF was in the range $25-43$ for hydrophobic silicone and $4.2-4.4$ for superhydrophobic coatings.

The surface asperities of the rough superhydrophobic surface may get damaged and removed by ice during deicing. Ensikat et al [31] have confirmed that rough surface asperities get indented into water and their tips can be damaged during the solidification of the liquid. The convex and concave structures can not only increase the contact area of ice and coating, 
but also make ice and coating embedded in each other forming anchoring effect which increases ice-adhesion strength tremendously. Hence the much higher ice-adhesion strength on the superhydrophobic coatings compared to those of the silicone based hydrophobic coatings with similar chemistry can be understood from the larger ice-solid contact areas of these samples.

\section{Conclusions}

The present study reveals that the hydrophilic surfaces like polymethylmethacrylate (PMMA) and polyurethane (PU) coatings displayed high ice-adhesion strength $(\tau)$ whereas hydrophobic silicone coatings had low ice-adhesion strength. It was found that the hydrophilic PU and PMMA coatings have high ice-adhesion strength of 820 and $1575 \mathrm{kPa}$, whereas the hydrophobic R2180 and RTV11 coatings had a low $\tau$ of $42 \pm 10 \mathrm{kPa}$ and $25 \pm 9$ $\mathrm{kPa}$ respectively. On the otherhand, superhydrophobic coatings showed higher $\tau$ values compared to hydrophobic coatings. It is observed that the work of adhesion follow the relation bare aluminium alloy $>$ PMMA $>$ PU $>$ R2180 $>$ RTV11 $>$ PMMA-HMS $>$ R2180EH5 > RTV11-EH5 indicating that superhydrophobic coatings had the lowest work of adhesion. The ice-adhesion reduction factor (ARF) for R2180 and RTV11 coatings were 25 and 43 times higher than on bare polished aluminium alloy $(\mathrm{ARF}=1)$. Thus maximum ARF was obtained for RTV11 coatings indicating good icephobic property. . Superhydrophobic silicone-composite coatings had lower ice-adhesion strength compared to PMMA-composite coatings due to the higher surface energy of PMMA. Surface morphology study of the samples by field emission scanning electron microscopy (FESEM) showed that hydrophilic and hydrophobic coatings were smooth and superhydrophobic coatings were rough with micro-bumps and nano-protrusions. It was concluded that smooth surface with low surface energy are responsible for low ice-adhesion strength. 
Acknowledgments: The authors are grateful to Mr. Shyam Chetty, Director, NAL and Dr. C. Anandan, Head, Surface Engineering Division, NAL Bangalore, for their constant support and encouragement of this work. We thank Mr. Siju and Mr. V. Praveen Kumar for recording FESEM and 3D roughness measurements. We greatly appreciate the funding assistance provided by CSIR network project for carrying out the studies. 


\section{References}

[1] J. L. Laforte, M.A. Allaire, J. Laflamme, Atmos. Res., 46 (1998) 143 - 158.

[2] V. K. Croutch, R. A. Hartley, J. Coatings Technol.,64 (1992) 41-53.

[3] T. F. Ford, O.D. Nichols, NRL Report 5832, 1962.

[4] J. Ayres, W.H. Simendinger, C. M. Balik, J. Coat. Technol. Res., 4 (2007) 463-471.

[5] A. J. Meuler, J. D.Smith, K. K. Varanasi, J. M. Mabry, G. H. McKinley, R. E. Cohen, ACS Appl. Mater. Interfaces 11 (2010) 3100-3110.

[6] A.J. Meuler, G. H. McKinley, R.E. Cohen, ACS Nano 4 (2011) 7048.

[7] S. L. Sivas, B. Riegler, R. Thomaier, K. Hoover, A silicone-based ice-phobic coating for aircraft, SAMPE 2007(www.nusil.com/whitepapers/index.aspx)

[8] R. Karmouch, G. G. Ross, Appl. Surf. Sci., 257 (2010) 665 - 669.

[9] H. Saito, K. Takai, G. Yamauchi, Surf. Coat. Int. 80 (1997) 168-171.

[10] L.L. Cao, A.K. Jones, V.K. Sikka, J.Z. Wu, D. Gao, Langmuir 25 (2009) 12444-12448.

[11] S.A. Kulinich, M. Farzaneh, Appl. Surf. Sci., 255 (2009) 8153-8157.

[12] S.A. Kulinich, M. Farzaneh, Cold Reg. Sci. Technol., 65 (2011) 60-64.

[13] R. Menini, M. Farzaneh, Surf. Coat. Technol., 203 (2009) 1941-1946.

[14] S.A. Kulinich, M. Farzaneh, Langmuir 25 (2009) 8854-8856.

[15] R. Menini, Z. Ghalmi, M. Farzaneh, Cold Reg. Sci. Technol., 65 (2011) 65-69.

[16] S. Farhadi, M. Farzaneh, S.A. Kulinich, Appl. Surf. Sci., 257 (2011) 6264-6269.

[17] P. Tourkine, M. Le Merrer, D. Quéré, Langmuir 25 (2009) 7214-7216.

[18] F.C. Wang, C.R. Li, Y.Z. Lv, F.C. Lv, Y.F. Du, Cold Reg. Sci. Technol., 62 (2010) 2933.

[19] L. Mishchenko, B. Hatton, V. Bahadur, J.A. Taylor, T. Krupenkin, J. Aizenberg, ACS Nano 4 (2010) 7699-7707 
[20] M. He, J.X. Wang, H.L. Li, X.L. Jin, J.J. Wang, B.Q. Liu, Y.L. Song, Soft Matter 6 (2010) 2396-2399

[21] A. Dotan, H. Dodiuk, C. Laforte, S. Kenig, J. Adhes. Sci. Technol., 23 (2009) 1907 1915.

[22] L. Yin, Q. Xia, J. Xue, S. Yang, Q. Wang, Q. Chen, Appl. Surf. Sci., 256 (2010) 67646769.

[23] G. Momen, M. Farzaneh, R. Jafari, Appl. Surf. Sci. 257 (2011) 6489-6493.

[24] K. K. Varanasi, T. Deng, J. D. Smith, M. Hsu, and N. Bhate, Appl. Phys. Lett. 97 (2010) 234102.

[25] S. Yang, Q. Xia, L. Zhu, J. Xue, Q. Wang, Q.-min Chen, Appl. Surf. Sci., 257 (2011) $4956-4962$.

[26] S.A. Kulinich, S. Farhadi, K. Nose, X.W. Du, Langmuir, 27 (2011) 25-29.

[27] M. F. Hassan, H. P. Lee, and S. P. Lim, Meas. Sci. Technol. 21, (2010) 075701 075709.

[28] M. Zou, S. Beckford, R. Wei, C. Ellis, G. Hatton, and M. A. Miller, Appl. Surf. Sci. 257 (2011) 3786 - 3792.

[29] J. Chen, J. Liu, M. He, K. Li, D. Cui, Q. Zhang, X. Zeng, Y. Zhang, J. Wang, Y. Song, Appl. Phys. Lett., 101 (2012) 111603 - 111605

[30] M. E. Schrader, Langmuir 11 (1995) 3585 - 3589

[31] J. J. Ensikat, A. J. Schulte, K. Koch, W. Brthlott, Langmuir 25 (2009) 13077 - 13083. 


\section{Table Captions}

Table 1 Water contact angle (WCA), sliding angle (SA) and work of adhesion (W) of the hydrophilic, hydrophobic and superhydrophobic coatings

Table 2. Ice adhesion strengths $(\tau)$ and Ice adhesion reduction factor (ARFs) of hydrophilic, hydrophobic and superhydrophobic coatings

Table 3. Average roughness $\left(\mathrm{R}_{\mathrm{a}}\right)$ and $\mathrm{Rms}$ roughness $\left(\mathrm{R}_{\mathrm{q}}\right)$ values obtained from the $3 \mathrm{D}$ roughness profiles of the coatings 


\section{Figure Captions}

Fig. 1. Photograph of ice adhesion tester based on zero degree cone method.

Fig. 2. Images of water droplet on (a) Bare AA, (b) PU, (c) RTV11 and (d) RTV11-EH5.

Fig. 3. Load versus displacement plots of (a) bare AA, (b) PU, (c) R2180 and (d) RTV11.

Fig. 4. Load versus displacement plots of superhydrophobic coatings, (a) R2180-CabEH5, (b)

RTV11-CabEH5 and (c) PMMA-HMS.

Fig. 5. FESEM images of (a, b) R2180, and (c,d) R2180-EH5 coatings; magnifications (a,c) $25.0 \mathrm{Kx} ;(\mathrm{b}, \mathrm{d}) 100.0 \mathrm{Kx}$.

Fig. 6. FESEM images of RTV11-EH5 coating; magnifications: (a) $10.0 \mathrm{Kx}$; (b) $50.0 \mathrm{Kx}$.

Fig.7. 3D roughness profiles of (a) PU, (b) PMMA, (c) R2180, (d) RTV11 hydrophobic coatings

Fig. 8. 3D roughness profiles (a) R2180-EH5, (b) RTV11-EH5, (c) PMMA-HMS

superhydrophobic coatings

Fig. 9. Ice adhesion reduction factor (ARF) of the studied coatings with reference to bare AA. 


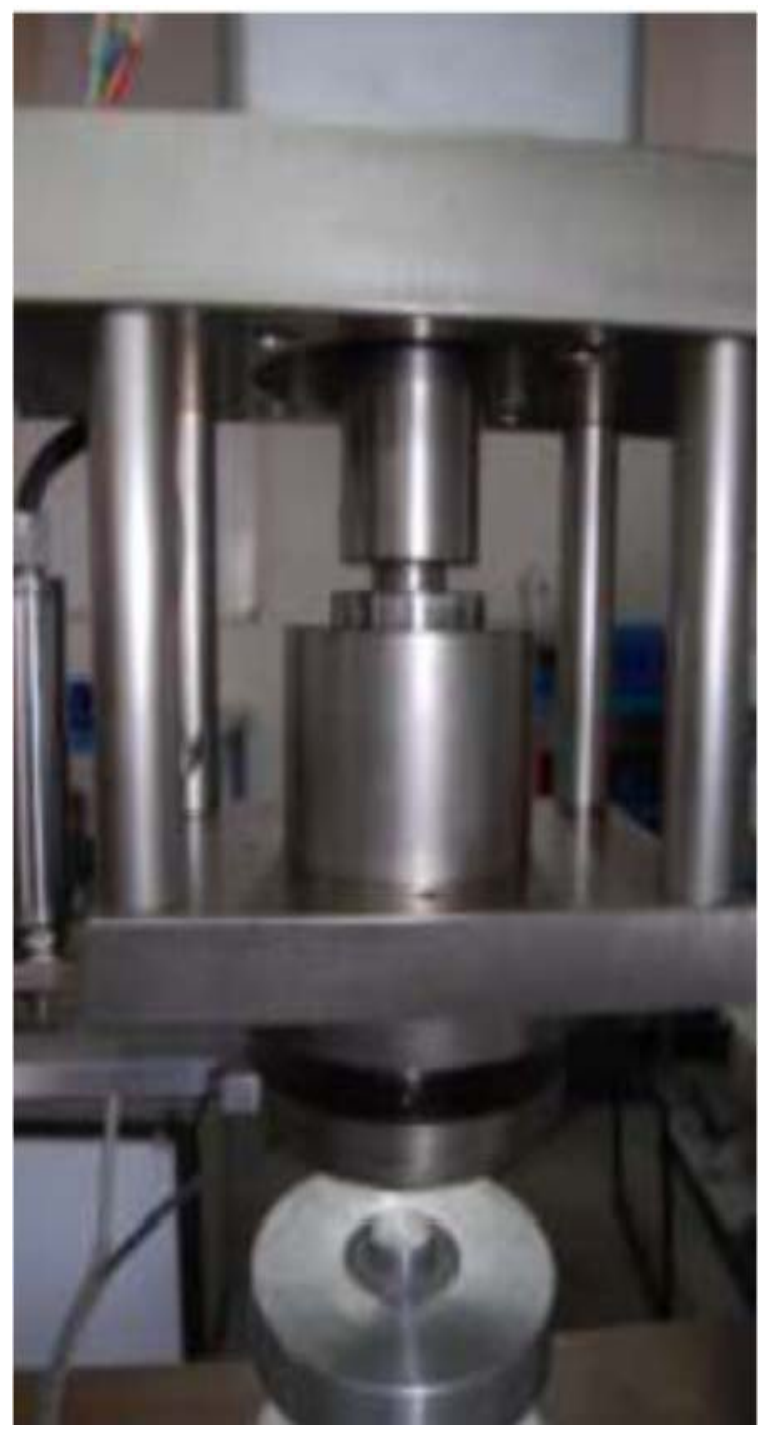

Fig. 1 


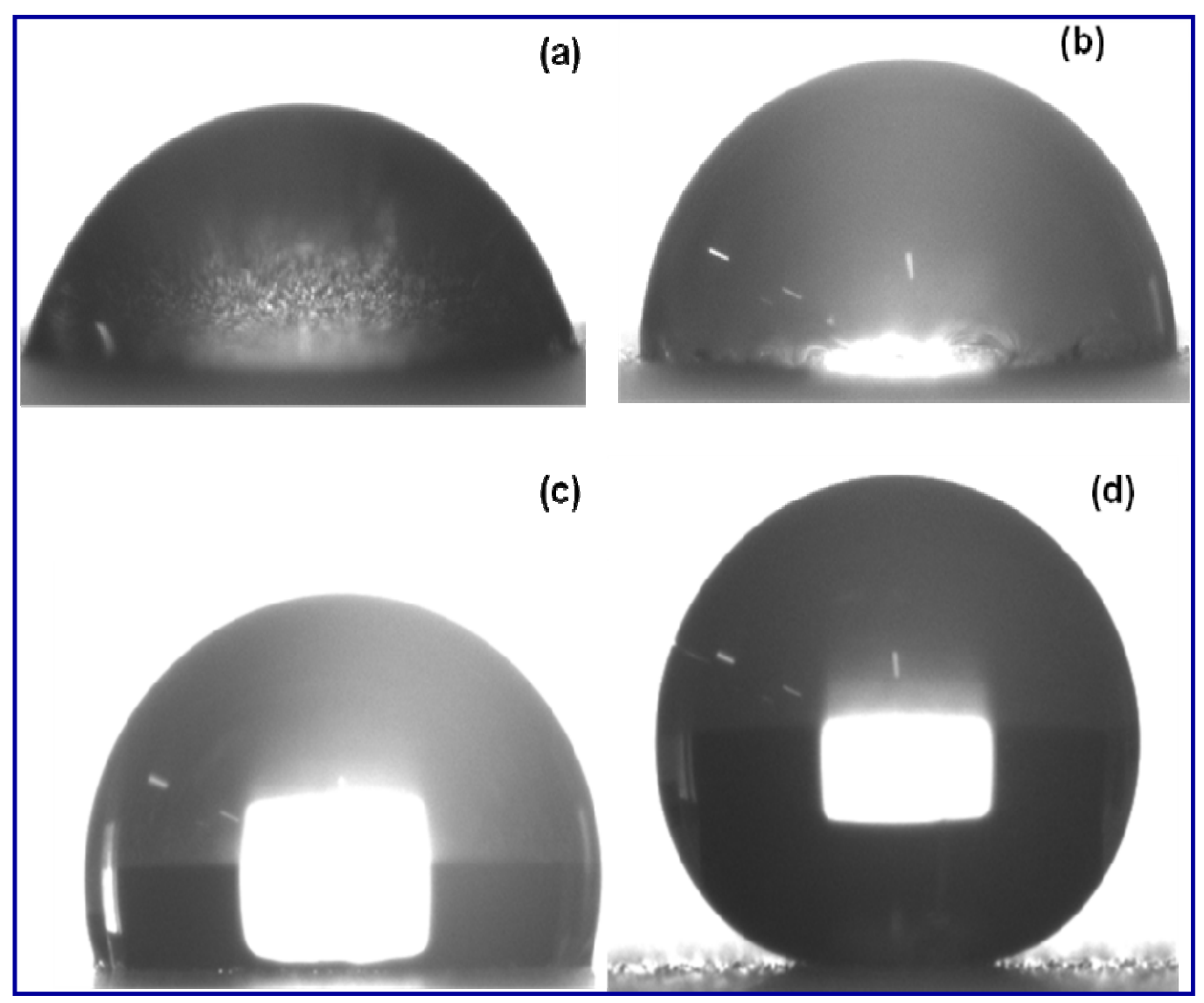

Fig. 2 

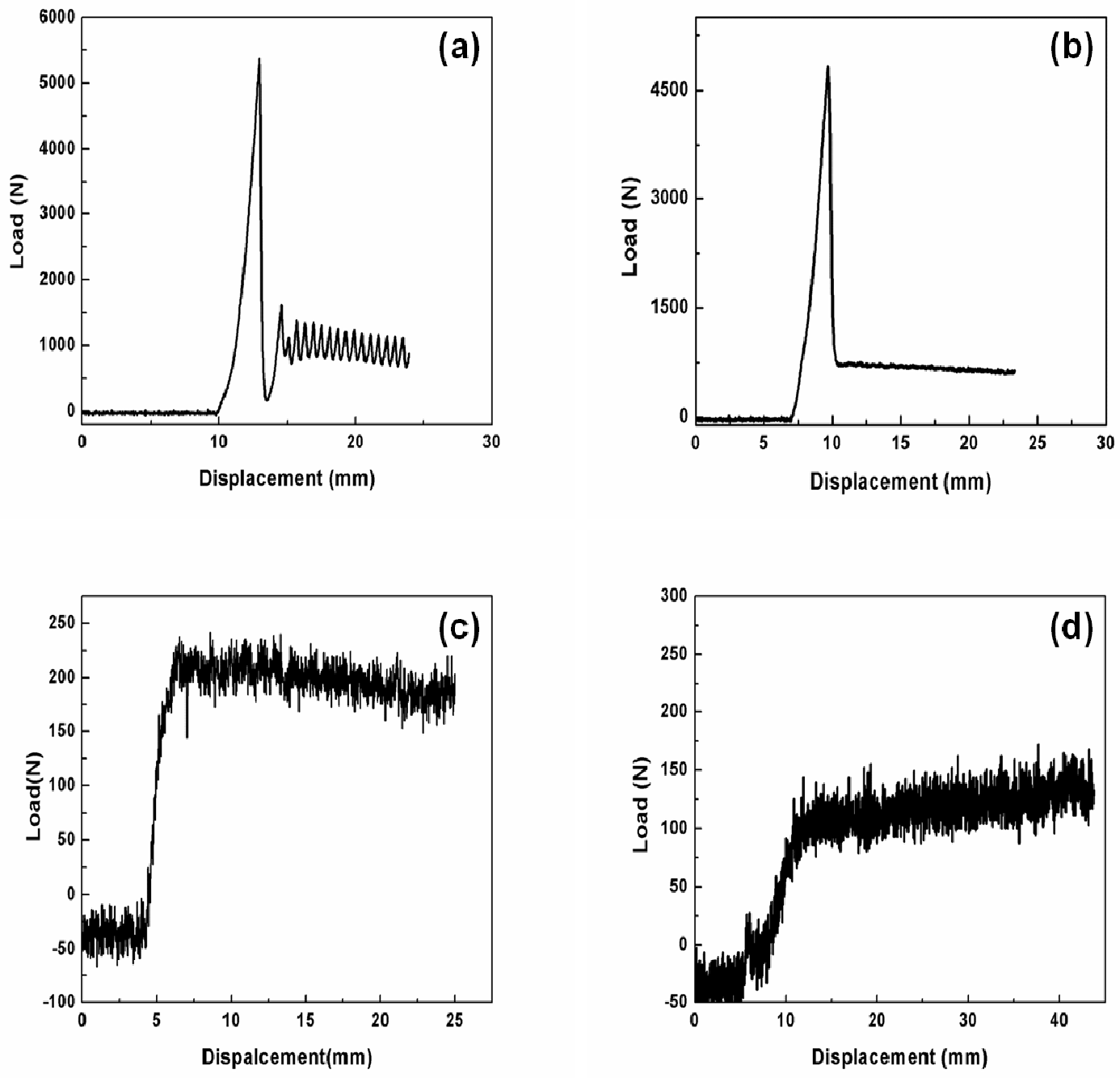

Fig. 3 

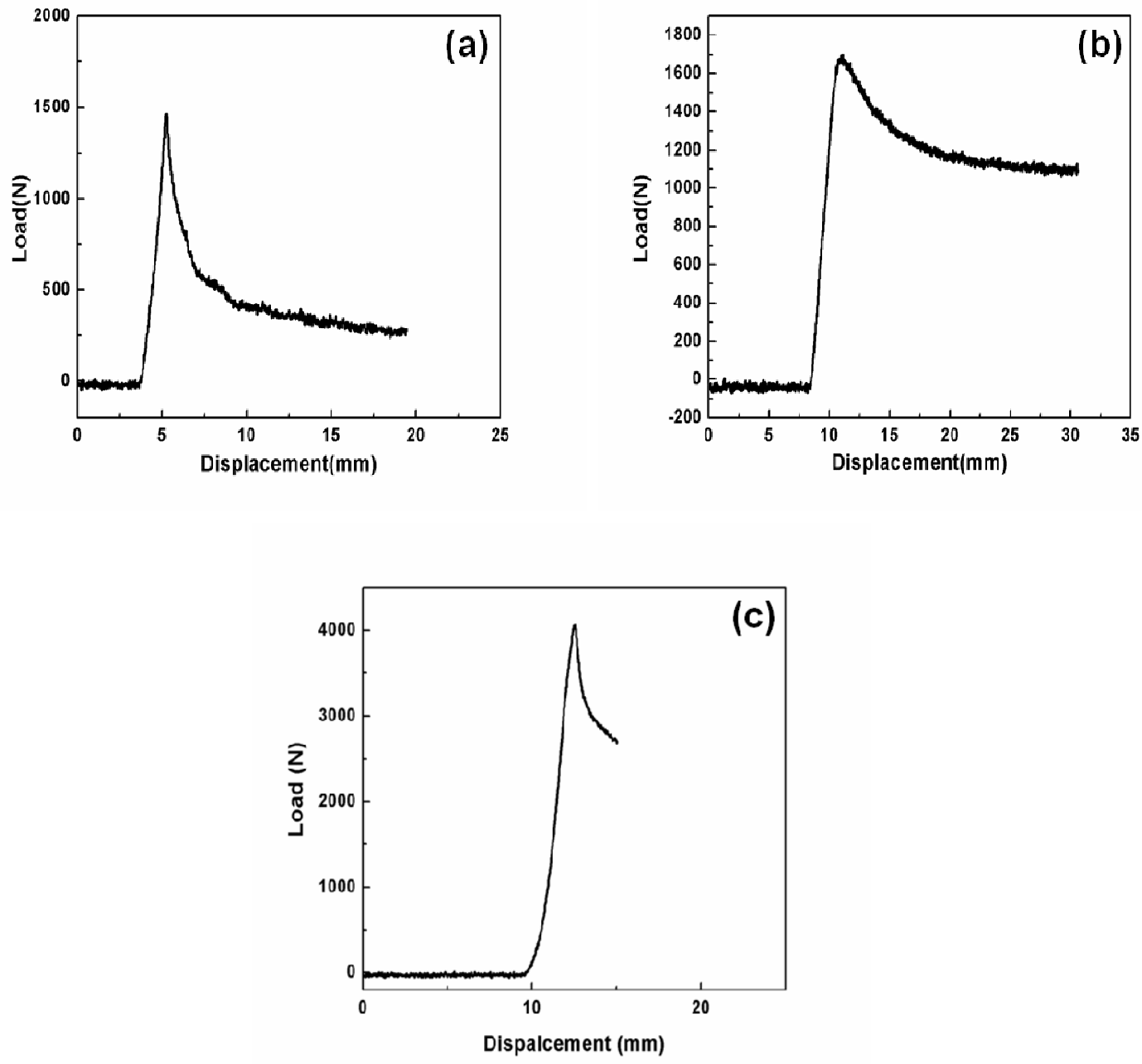

Fig. 4 


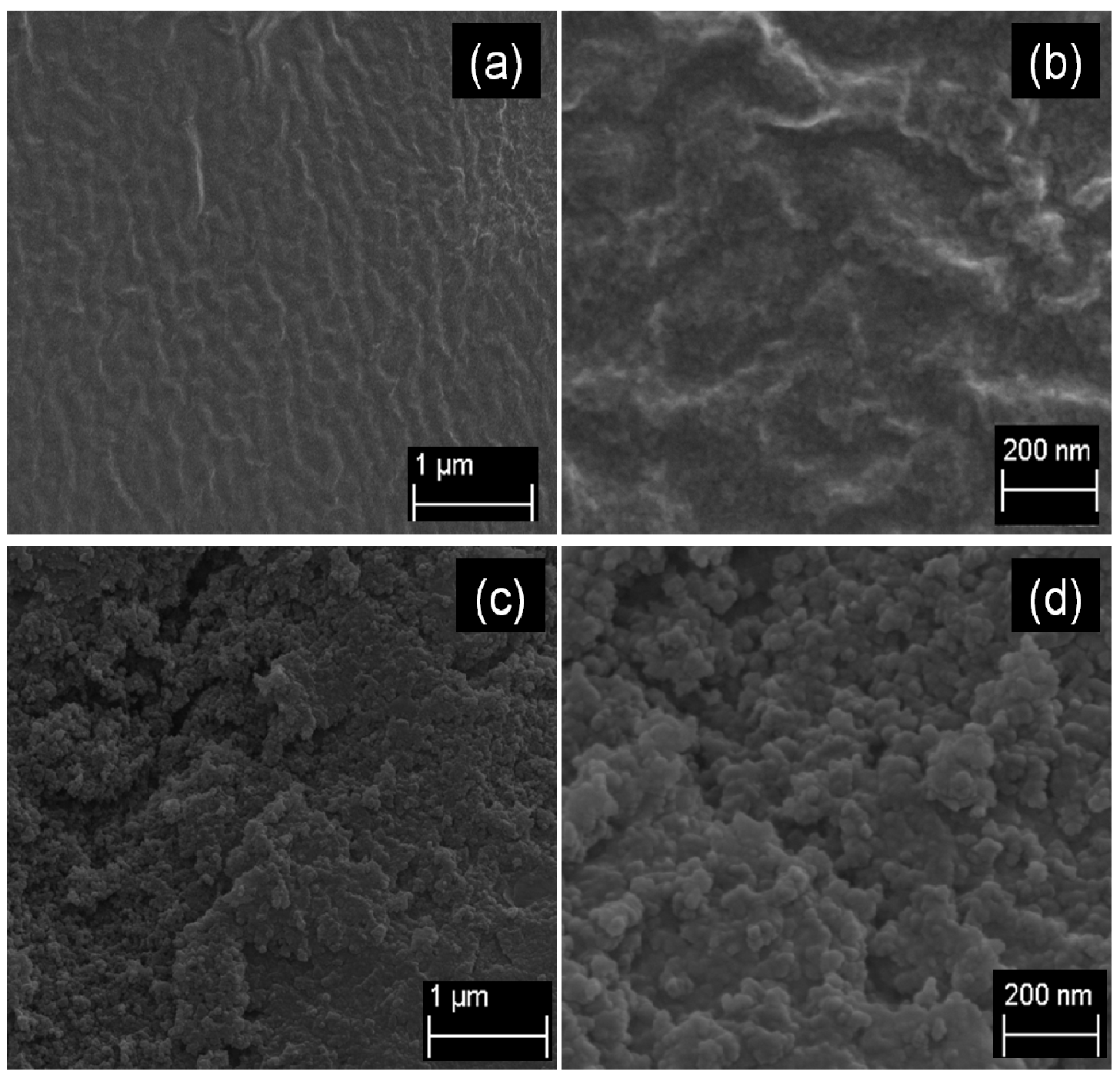

Fig. 5 


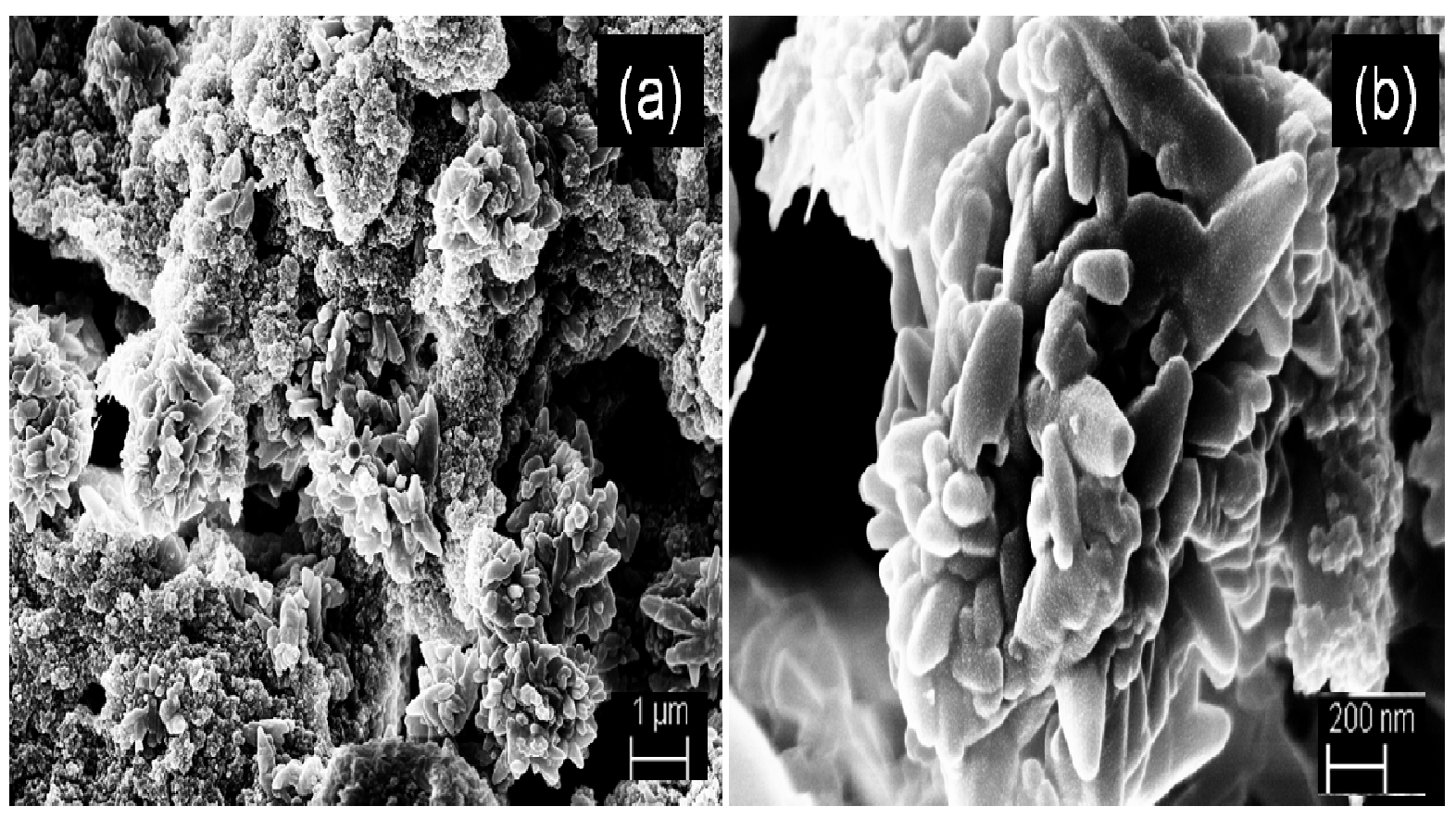

Fig. 6 

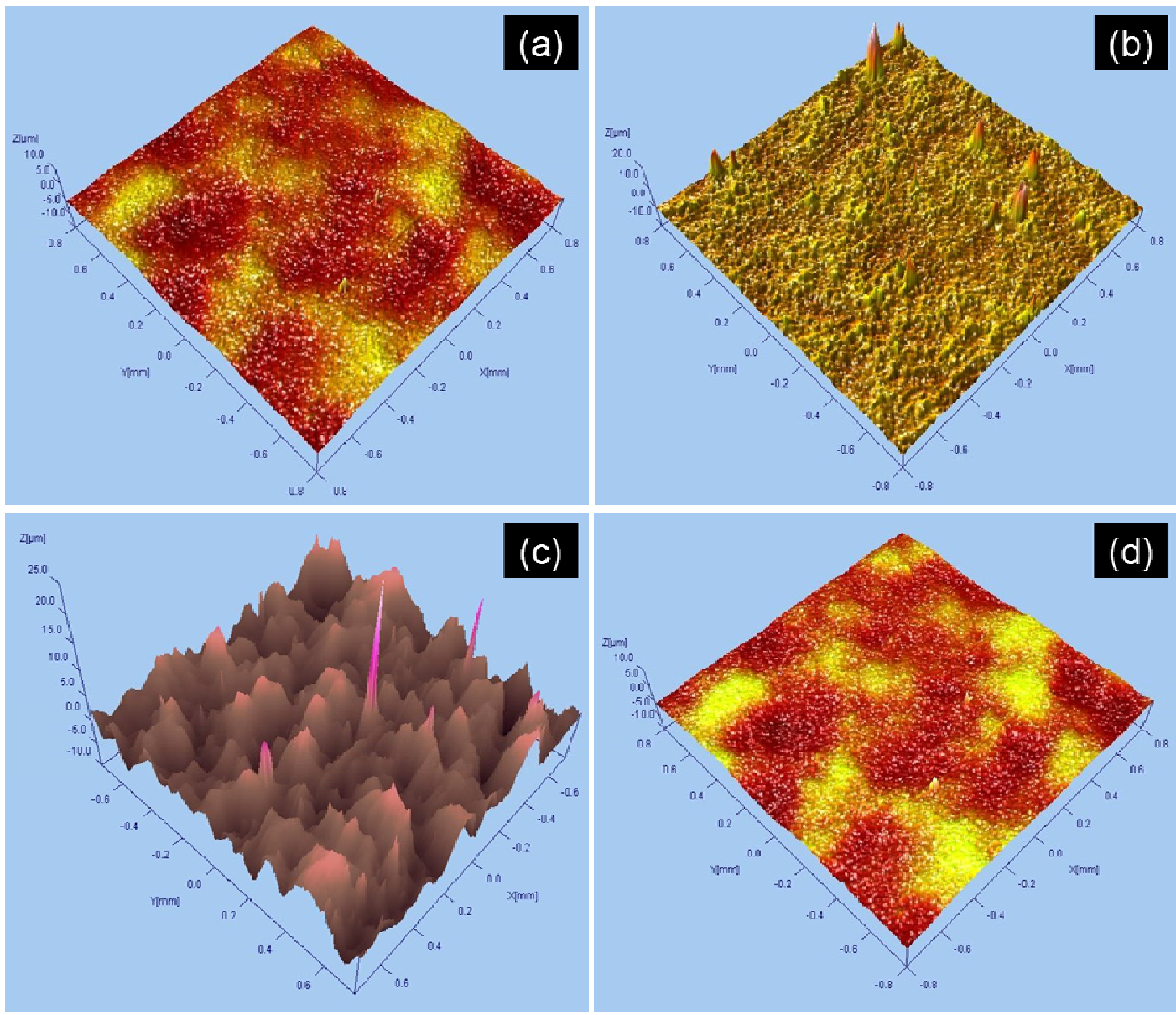

Fig. 7 


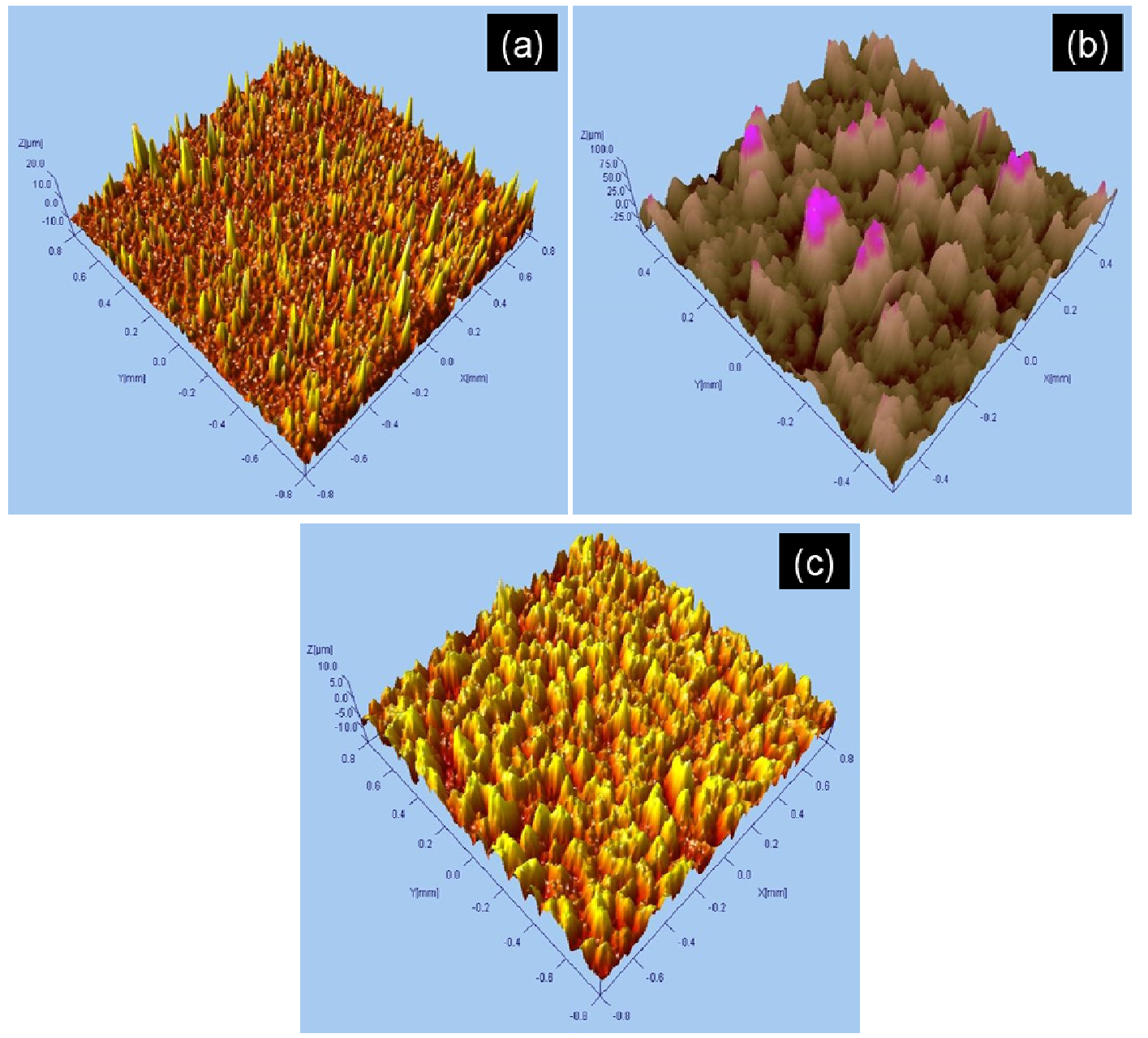

Fig. 8 


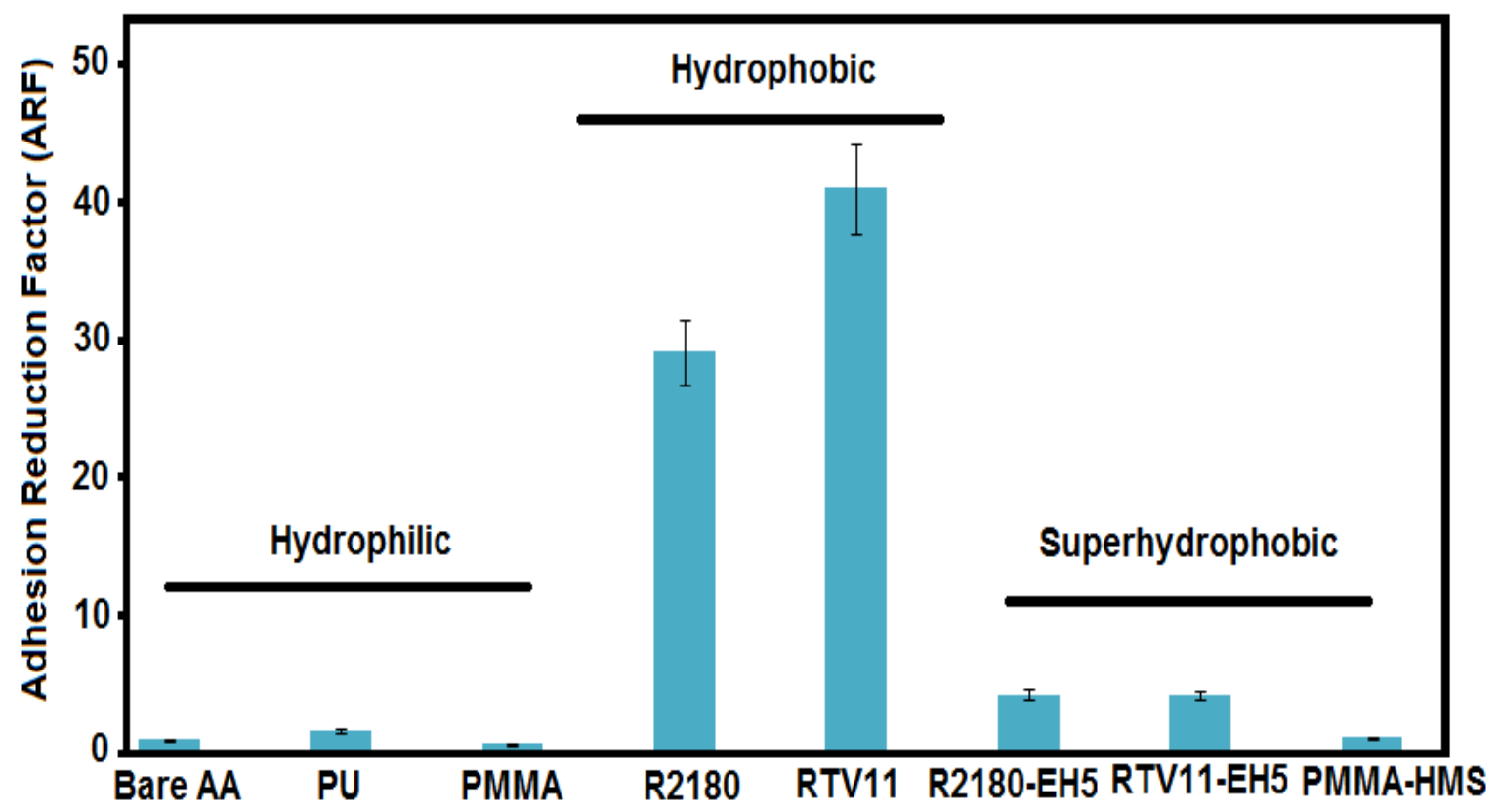

Fig. 9 
Table 1

Water contact angle (WCA), sliding angle (SA) and work of adhesion (W) of the hydrophilic, hydrophobic and superhydrophobic coatings

\begin{tabular}{llll}
\hline Sample/ & WCA $\left(^{\circ}\right)$ & SA $\left(^{\circ}\right)$ & Work of adhesion \\
coating & & & $(\mathrm{mN} / \mathrm{m})$ \\
\hline Bare AA & 67 & $>90$ & 101.80 \\
PU & 77 & $>90$ & 89.67 \\
PMMA & 73 & $>90$ & 94.60 \\
R2180 & 103 & $>90$ & 56.73 \\
RTV 11 & 105 & $>90$ & 54.25 \\
R2180-EH5 & 155 & $<2$ & 6.86 \\
RTV11-EH5 & 158 & $<2$ & 5.33 \\
PMMA-HMS & 153 & $<2$ & 7.98 \\
\hline
\end{tabular}


Table 2. Ice adhesion strengths $(\tau)$ and Ice adhesion reduction factor (ARFs) of hydrophilic, hydrophobic and superhydrophobic coatings

\begin{tabular}{l|l|l}
\hline Sample & $\begin{array}{l}\text { Average ice adhesion strength } \\
(\mathrm{kPa})\end{array}$ & $\begin{array}{l}\text { Ice adhesion } \\
\text { reduction factor } \\
\text { ARF }\end{array}$ \\
\hline Bare AA & $\begin{array}{l}1072 \pm 120 \\
820 \pm 40\end{array}$ & 1.0 \\
PU & $1535 \pm 240$ & 1.31 \\
PMMA & $42.5 \pm 15$ & $<1.0$ \\
R2180 & $24.8 \pm 8$ & 25.2 \\
RTV11 & $258 \pm 25$ & 43.2 \\
R2180-EH5 & $243 \pm 20$ & 4.16 \\
RTV11-EH5 & $799 \pm 90$ & 1.34 \\
PMMA-HMS & & \\
\hline
\end{tabular}


Table 3. Average roughness $\left(R_{a}\right)$ and $R m s$ roughness $\left(R_{q}\right)$ values obtained from the $3 \mathrm{D}$ roughness profiles of the coatings

\begin{tabular}{l|l|l}
\hline Sample & $\begin{array}{l}\text { Average roughness }\left(\mathrm{R}_{\mathrm{a}}\right) \\
(\mu \mathrm{m})\end{array}$ & $\begin{array}{l}\text { Rms roughness }\left(\mathrm{R}_{\mathrm{q}}\right) \\
(\mu \mathrm{m})\end{array}$ \\
\hline Bare AA & 0.30 & 0.368 \\
PU & 0.39 & 0.475 \\
PMMA & 0.83 & 1.652 \\
R2180 & 2.21 & 2.771 \\
RTV11 & 0.38 & 0.485 \\
R2180-EH5 & 1.62 & 1.939 \\
RTV11-EH5 & 4.46 & 12.12 \\
PMMA-HMS & 1.99 & 2.448 \\
\hline
\end{tabular}

